\title{
Milliliter per Hour per Milligram per Kilogram
}

National Cancer Institute

\section{Source}

National Cancer Institute. Milliliter per Hour per Milligram per Kilogram. NCI Thesaurus.

Code C120778.

A unit of concentration equal to milliliter per hour divided by milligram per kilogram. 